\title{
Validação de método analítico espectrofotométrico UV para determinação de ácido úsnico em lipossomas
}

\author{
Marigilson Pontes de Siqueira-Moura ${ }^{1,2}$, Mariane Cajubá Britto Lira ${ }^{1,2}$, \\ Nereide Stela Santos-Magalhães ${ }^{2,3 *}$ \\ ${ }^{1}$ Departamento de Ciências Farmacêuticas, Universidade Federal de Pernambuco, \\ ${ }^{2}$ Laboratório de Imunopatologia Keizo-Asami (LIKA), Universidade Federal de Pernambuco, ${ }^{3}$ Departamento de \\ Bioquímica, Universidade Federal de Pernambuco
}

*Correspondência:

N. S. S. Magalhães

Laboratório de Imunopatologia KeizoAsami (LIKA)

Universidade Federal de Pernambuco Av. Prof. Moraes Rego, 1235, Cidade Universitária

50670-901, Recife - PE, Brasil.

E-mail: nssm@ufpe.br
O ácido úsnico (AU) é um composto de origem liquênica e tem demonstrado importantes atividades biológicas, tais como: antitumoral, antimicrobiano, antiviral, antiproliferativo e antiinflamatório. Os lipossomas são vesículas lipídicas contendo espaço aquoso interno e têm sido utilizados como carreadores coloidais de fármacos, principalmente na terapêutica de câncer e infecções bacterianas e fúngicas. $O$ objetivo desse trabalho foi desenvolver e validar um método espectrofotométrico UV para determinação de ácido úsnico em lipossomas. Os parâmetros de validação linearidade, precisão, exatidão, robustez, limites de deteç̧ão e quantificação foram determinados segundo diretrizes internacionais de padronização e Farmacopéia Americana. A faixa de linearidade foi de 3 a $15 \mu \mathrm{g} \cdot \mathrm{mL}^{-1}$, a equação de regressão: absorbância $=0,070 \times[\mathrm{AU}]\left(\mu \mathrm{g} \cdot \mathrm{mL}^{-1}\right)+0,013$ e $r=0,9997 . \mathrm{A}$ repetibilidade (coeficiente de variação) do método foi 1,96\% e a precisão intermediária indicou que a diferença entre as médias foi estatisticamente insignificante $(P<0,05)$. A exatidão revelou média percentual de recuperação de 100,4\%. O método foi robusto apesar da variação de temperatura e solventes. Os limites de detecção e quantificação do ácido úsnico foram de 0,34 e $1,13 \mu \mathrm{g} . \mathrm{mL}^{-1}$, respectivamente. $O$ doseamento do ácido úsnico nos lipossomas foi de $96,8 \%( \pm 0,2)$. O método proposto é exato, preciso e reprodutivel sendo capaz de quantificar o ácido úsnico em matéria-prima e em preparações farmacêuticas.
Unitermos

- Ácido úsnico/determinação

- Lipossomas

- Espectrofotometria no ultravioleta

- Análise quantitativa/ validação de método

\section{INTRODUÇÃO}

O ácido úsnico [2,6-diacetil-7,9-dihidroxi-8,9bdimetil-1,3-(2H,9H)-dibenzofurano] (Figura 1) é um composto derivado do metabolismo secundário liquênico e de- sempenha importante papel biológico, conferindo aos liquens proteção contra microrganismos invasores, raios UV e ressecamento (Cocchietto et al., 2002). Este derivado liquênico apresenta-se, na forma cristalina, com coloração levemente amarelada e existe na natureza em duas for- 
mas enantioméricas: $(+)$ e (-)-ácido úsnico, dependendo da posição do grupo metila do carbono quiral $9 \mathrm{~b}$ (Ingólfsdóttir, 2002). O ácido úsnico é praticamente insolúvel em água $\left(0,01 \mathrm{~g} / 100 \mathrm{~mL}\right.$ a $25^{\circ} \mathrm{C}$, Merck Index, 1995) e solúvel em solventes orgânicos, tais como: dimetilsulfóxido, acetona, clorofórmio, metanol, acetato de etila e diclorometano (Ingólfsdóttir, 2002). O ácido úsnico apresenta atividade antimicrobiana (Lauterwein et al., 1995), em particular contra Streptococcus mutans, agente etiológico de cáries e periodontites (Grasso et al., 1989).

O ácido úsnico, como agente antimicrobiano, é constituinte de diversas preparações farmacêuticas, tais como: dentifrício (Grasso et al., 1989), colutório (Ferrarri et al., 1988; Ghione et al., 1988; Lodetti et al., 2000), óvulos vaginais (Scirpa et al., 1999), antitranspirante e desodorante (Clothier et al., 2002; Elliott et al., 2005); em cápsulas como suplemento dietético - LipoKinetix ${ }^{\circledR}$ (Neef et al., 2004), atualmente fora do mercado devido à hepatoxicidade; em microesferas (Ribeiro-Costa et al., 2004) e nanocápsulas (Santos et al., 2005) como antineoplásico.

Métodos de doseamento do ácido úsnico utilizando cromatografia líquida de alta eficiência (CLAE) com detecção UV são descritos para doseamento de matériaprima e produtos farmacêuticos - cápsulas, comprimidos e preparações líquidas (Ji, Khan, 2005), microesferas e nanocápsulas (Ribeiro-Costa et al., 2004; Santos et al., 2005) e em plasma (Venkataramana, Krishna, 1992).

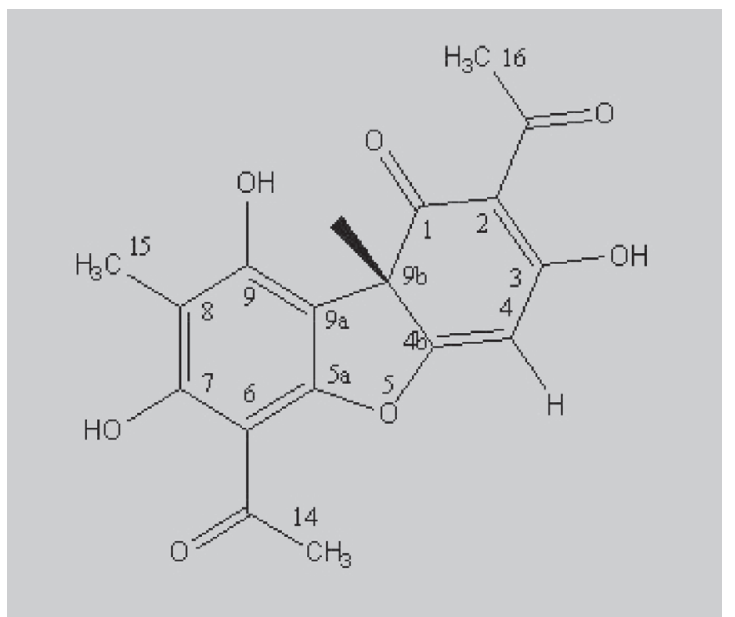

FIGURA 1 - Estrutura química do ácido úsnico $\left(\mathrm{C}_{18} \mathrm{H}_{16} \mathrm{O}_{7}\right)$.

Apesar das vantagens indiscutíveis na utilização da técnica cromatográfica CLAE, esta apresenta algumas limitações como o alto custo da instrumentação e da operação, tempo relativamente longo de análise e ainda a necessidade de experiência no manuseio do equipamento e tra- tamento de amostras. A análise do teor de ativos utilizando espectroscopia com detecção em ultravioleta (UV) é menos onerosa, mais facilmente exeqüível no controle de qualidade de matéria-prima e produtos farmacêuticos, além de estudos de cinética de liberação de fármacos.

A quantificação do ácido úsnico como matéria-prima e em produtos farmacêuticos carece do desenvolvimento e validação de um método analítico, utilizando espectroscopia UV para controle de qualidade, o qual possa ser perfeitamente adaptado à rotina de análises de matéria-prima e produto acabado.

Neste cenário, o objetivo do presente estudo é desenvolver e validar um método analítico para quantificar o ácido úsnico como matéria-prima e em formas farmacêuticas através de espectrofotometria UV. A aplicação do método foi efetuada no doseamento do ácido úsnico em formas lipossomais.

\section{MATERIAL E MÉTODOS}

\section{Reagentes e amostras}

Fosfatidilcolina de soja (SjPC) (Epikuron ${ }^{\circledR} 200$, 98\%) foi obtida da Lucas Meyer (Hamburgo, Alemanha). Colesterol, estearilamina e (+)-ácido úsnico (com grau de pureza de 98\%) foram adquiridos na Sigma-Aldrich (St. Louis, EUA). Acetonitrila, clorofórmio, metanol e fosfato monobásico de potássio foram fornecidos pela Merck (Darmstadt, Alemanha). Os lipossomas contendo ácido úsnico foram produzidos com fosfatidilcolina, colesterol e estearilamina pelo método de hidratação do filme lipídico, seguido de sonicação (Lasic, 1993). Todas as análises quantitativas de doseamento do ácido úsnico foram realizadas em espectrofotômetro UV-Vis Ultrospec ${ }^{\circledR} 3000$ Pro (Amersham Pharmacia Biotech), utilizando células de quartzo de $10 \mathrm{~mm}$ de caminho óptico.

\section{Desenvolvimento de método analítico espectrofotométrico}

O comprimento de onda de maior absorbância do ácido úsnico foi determinado em espectrofotômetro UV-Vis entre os comprimentos de onda de 225 e $400 \mathrm{~nm}$ de diluição adequada da solução estoque padrão em metanol.

\section{Solução padrão de ácido úsnico}

A solução estoque padrão foi obtida pesando exatamente cerca de $20 \mathrm{mg}$ de ácido úsnico, que foram transferidas para balão volumétrico de $50 \mathrm{~mL}$ e solubilizado em mistura de acetonitrila e metanol 1:5,25 
( $8 \mathrm{~mL}$ de acetonitrila e $42 \mathrm{~mL}$ de metanol), e então, submetida à sonicação por 5 minutos. Esta solução foi aquecida em banho-maria a $37^{\circ} \mathrm{C}$ até completa solubilização do ácido úsnico durante 5 minutos. Por fim, após resfriamento da solução à temperatura ambiente, o volume foi completado com metanol. A concentração final de ácido úsnico foi de $0,4 \mathrm{mg} \cdot \mathrm{mL}^{-1}$. Diluições apropriadas da solução estoque padrão foram efetuadas com metanol para execução do estudo de validação.

\section{Preparação da amostra de ácido úsnico na forma lipossomal}

Para determinação do ácido úsnico transferiu-se alíquota da forma lipossomal equivalente a $60 \mathrm{mg}$ do analito para balão volumétrico de $5 \mathrm{~mL}$. Em seguida, adicionou-se à amostra $40 \mathrm{~mL}$ de metanol e $40 \mathrm{~mL}$ de clorofórmio. A solução foi submetida à sonicação por 5 minutos para romper a estrutura lamelar dos lipossomas e liberar o conteúdo em ácido úsnico encapsulado. Por fim, o volume da solução foi completado com metanol. A concentração teórica de ácido úsnico, na amostra, foi de $12 \mathrm{mg} \mathrm{mL}^{-1}$. As amostras foram filtradas através de membranas de celulose de poros de 0,45 mm (Millipore, EUA). Os ensaios de doseamento foram realizados em triplicata.

\section{Validação do método analítico}

A validação de um método analítico é definida como o processo pelo qual é estabelecida, por estudos laboratoriais, que as características de eficiência do método correspondem aos requerimentos necessários à aplicação analítica desejada (Pérez-Lozano et al., 2004). Os parâmetros de validação linearidade, precisão, exatidão, robustez, limite de detecção (LD) e limite de quantificação (LQ) foram avaliados segundo os critérios preconizados pela Conferência Internacional de Padronização de Requerimentos Técnicos para Registro de Produtos Farmacêuticos de Uso Humano (International Conference on Harmonisation-ICH of Technical Requirements for Registration of Pharmaceuticals for Human Use, ICH, 2005) e pela Farmacopéia Americana (USP, 2003).

\section{Linearidade}

A linearidade foi estabelecida pela média de nove curvas padrão autênticas, as quais foram obtidas em sete níveis de concentrações diferentes de ácido úsnico: 3, 5, 6, $8,10,12$ e $15 \mathrm{mg} \cdot \mathrm{mL}^{-1}$. A faixa de variação da concentração corresponde de 30 a $150 \%$ da concentração teste. Cada concentração foi determinada em triplicata para cada cur- va padrão $(n=27)$. A linearidade foi avaliada através de análise de regressão linear, utilizando ajuste dos dados pelo método dos mínimos quadrados. Para avaliar numericamente a qualidade do ajuste do modelo, utilizou-se a análise de variância (ANOVA) com teste unilateral, $\mathrm{p}<0,05$.

\section{Precisão}

Para o estudo de precisão foi utilizada a concentração de $10 \mathrm{mg} \cdot \mathrm{mL}^{-1}$ do ácido úsnico (concentração teste) determinada em soluções diferentes $(n=6)$. As análises foram realizadas em dois dias diferentes, por dois analistas diferentes. As médias dos resultados obtidos foram avaliadas pelo teste $t$ de Student (teste bilateral, $\mathrm{p}<0,05$ ).

\section{Exatidão}

A exatidão foi avaliada pelo método de recuperação do analito adicionado em quantidades conhecidas à formulação lipossomal placebo em três concentrações diferentes: 5, 10 e 15 mg.mL ${ }^{-1}$, correspondendo, respectivamente, a 50,100 e $150 \%$ da concentração teste. Todas as amostras x $150 \%$ foram preparadas em triplicata $(n=9)$. O coeficiente de variação e a porcentagem de recuperação foram utilizados para avaliar a exatidão definida como:

Exatidão $=($ concentração experimental obtida/concentração teórica) $\times 100$

\section{Robustez}

A robustez do método proposto foi verificada pela variação de temperatura de análise $\left(4{ }^{\circ} \mathrm{C}\right.$ e $\left.25^{\circ} \mathrm{C}\right)$ e pela mudança de fabricante do metanol (Merck e OmniSolv - EMD $\left.{ }^{\mathrm{TM}}\right)$. A concentração de $10 \mathrm{mg} \cdot \mathrm{mL}^{-1}$ de ácido úsnico foi determinada em seis ensaios diferentes. A avaliação da robustez foi realizada pela análise dos coeficientes de variação entre as médias obtidas, utilizando o teste $t$ de Student (teste bilateral, $\mathrm{p}<0,05$ ).

\section{Limite de Detecção (LD) e Limite de Quantificação (LQ)}

Os limites de detecção (a) e quantificação (b) foram determinados, matematicamente, a partir da curva analítica resultante da média das três curvas analíticas. O cálculo para determinar os valores correspondentes ao LD e LQ, baseia-se no desvio padrão do residual da linha de regressão e sua relação com a inclinação da reta (coeficiente angular) na curva analítica, seguindo as relações:

$L D=(D . P . / I) \times 3,3$ 
Onde,

D.P.: desvio padrão do intercepto com relação ao eixo dos Y; I: valor da inclinação da curva analítica.

\section{RESULTADOS E DISCUSSÃO}

\section{Desenvolvimento do método analítico}

O espectro de varredura do ácido úsnico em metanol revelou absorbância máxima no comprimento de onda de $280 \mathrm{~nm}$ (Figura 2), resultado este de acordo com relatos prévios (Takani et al., 2002).

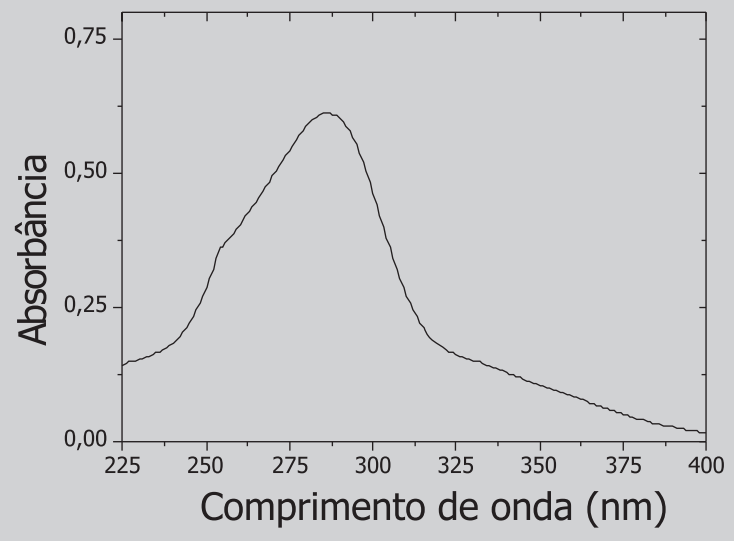

FIGURA 2 - Espectro de varredura do ácido úsnico $\left(\lambda_{\text {máx }} 280 \mathrm{~nm}\right)$ em metanol.

\section{Linearidade}

Os dados da curva analítica, resultante da média de nove curvas padrão, foram ajustados por análise de regressão linear (Tabela I), cuja equação da reta é dada por: Absorbância $=0,070 \times$ x AU] $\left(\mu \mathrm{g} \cdot \mathrm{mL}^{-1}\right)+0,013$. O coeficiente de correlação foi de 0,9997, significando que 99,97 \% da variação total em torno da média é explicada pela regressão linear, com os resíduos (erro) de apenas 0,03 \%.
O teste unilateral de análise de variância (ANOVA) avaliou a qualidade do ajuste do modelo linear. A análise dos dados da linearidade demonstrou ser a regressão altamente significativa, bem como não foi evidenciada a falta de ajuste do modelo, uma vez que, os valores de F calculados foram menores do que os valores de $\mathrm{F}$ críticos no nível de $95 \%$ de confiança (Tabela II). Portanto, o método analítico desenvolvido possui faixa de linearidade entre as concentrações de ácido úsnico de 3 e $15 \mathrm{mg} \cdot \mathrm{mL}^{-1}$.

TABELA I - Curva analítica do ácido úsnico

\begin{tabular}{lcc}
\hline $\begin{array}{l}\text { Concentração de } \\
\begin{array}{l}\text { ácido úsnico } \\
\left(\mu \mathrm{g} . \mathrm{mL}^{-1}\right)\end{array}\end{array}$ & $\begin{array}{c}\text { Valor médio de } \\
\text { absorbância } \\
( \pm \mathrm{D} . P .)^{*}\end{array}$ & $\begin{array}{c}\text { Concentração } \\
\text { calculada de } \\
\text { ácido úsnico } \\
( \pm \mathrm{D} . \mathrm{P} .)^{*}\end{array}$ \\
\hline 3 & $0,219 \pm 0,008$ & $2,95 \pm 0,11$ \\
5 & $0,369 \pm 0,015$ & $5,08 \pm 0,21$ \\
6 & $0,438 \pm 0,019$ & $6,07 \pm 0,26$ \\
8 & $0,578 \pm 0,029$ & $8,07 \pm 0,41$ \\
10 & $0,734 \pm 0,015$ & $10,29 \pm 0,21$ \\
12 & $0,855 \pm 0,020$ & $12,02 \pm 0,29$ \\
15 & $1,069 \pm 0,033$ & $15,08 \pm 0,46$ \\
\hline
\end{tabular}

*D.P. $=$ desvio padrão, $\mathrm{n}=27$.

\section{Precisão}

A precisão foi avaliada pelos estudos de repetibilidade e precisão intermediária. A repetibilidade revelou coeficiente de variância de $1,96 \%$, portanto, menor do que o valor máximo exigido de $5 \%$ (Tabela III). A precisão intermediária, que avalia a variação de ensaios realizados em dias e com analistas diferentes, mostrou valor de desvio padrão relativo de $1,89 \%$. Através do teste $t$ de Student foi avaliada a possível existência de diferença estatisticamente significativa entre as médias obtidas para os ensaios realizados em dias diferentes e com analistas diferentes. Como pode ser observado na Tabela IV, em todos os casos o $t$

TABELA II - Análise de variância para linearidade do método de doseamento do ácido úsnico em espectroscopia UV

\begin{tabular}{lccccc}
\hline Fonte de Variação & Soma Quadrática & Graus de Liberdade & Média Quadrática & \multicolumn{2}{c}{ Valores de F } \\
& & & & $\mathrm{F}_{\text {calculado }}$ & $\mathrm{F}_{\text {crítico }}$ \\
\hline Regressão & 4,796892 & 1 & 4,796892 & $10.015,42$ & $4,00^{\mathrm{a}}$ \\
Resíduos & 0,029216 & 61 & 0,000478 & & \\
Falta de ajuste & 0,003645 & 5 & 0,000729 & 1,60 & $2,37^{\mathrm{b}}$ \\
Erro puro & 0,025571 & 56 & 0,000456 & & \\
Total & 4,826108 & 62 & & \\
\hline
\end{tabular}

${ }^{a}$ Valor teórico de $\mathrm{F}(1,61)$ baseado no teste ANOVA unilateral $(\mathrm{p}<0,05)$.

${ }^{b}$ Valor teórico de $\mathrm{F}(5,56)$ baseado no teste ANOVA unilateral $(\mathrm{p}<0,05)$. 
calculado foi sempre menor do que o $t$ crítico, demonstrando não existir diferença estatisticamente significativa entre as médias, ou seja, os resultados obtidos expressam o mesmo valor $(\mathrm{p}<0,05)$.

TABELA III - Resultados do estudo de repetibilidade do método de dosagem do ácido úsnico em espectroscopia UV

\begin{tabular}{lcc}
\hline Dados Estatísticos & $\begin{array}{c}\text { Absorbância } \\
(280 \mathrm{~nm})\end{array}$ & $\begin{array}{c}\text { Concentração } \\
\left(\mu \mathrm{g} \cdot \mathrm{mL}^{-1}\right)\end{array}$ \\
\hline & 0,722 & 10,12 \\
& 0,707 & 9,91 \\
& 0,721 & 10,11 \\
& 0,713 & 10,00 \\
& 0,740 & 10,38 \\
Média & 0,741 & 10,40 \\
D.P. & 0,724 & 10,15 \\
C.V. (\%) & 0,014 & 0,200 \\
\hline
\end{tabular}

*D.P. $=$ desvio padrão; C.V.= coeficiente de variação.

\section{Exatidão}

A exatidão foi verificada para três níveis de concentração: baixa, média e alta. Os dados experimentais obtidos revelaram média de recuperação do analito de 100,4 \% (100,3-100,8\%) e que o maior coeficiente de variação foi de cerca de 3,41 \% (Tabela V). Os resultados do estudo de exatidão demonstram que pequenas variações da concentração de ácido úsnico, podem ser prontamente quantificadas pelo método, bem como não há interferência dos excipientes da forma lipossomal no doseamento do produto final, portanto, o método analítico desenvolvido é suficientemente exato.

\section{Robustez}

A variação da temperatura de análise não afetou a absorbância do ácido úsnico. As concentrações médias obtidas para as análises de soluções acondicionadas nas
TABELA IV - Dados estatísticos do teste $t$ de Student para a precisão intermediária do método de dosagem do ácido úsnico em espectroscopia UV

\begin{tabular}{|c|c|c|c|c|}
\hline \multicolumn{5}{|c|}{ Valores de $\mathrm{t}$} \\
\hline \multicolumn{2}{|c|}{ Inter-dia } & Intr: & & \multirow[t]{2}{*}{$\begin{array}{c}\mathrm{t}_{\text {crítico }} \\
\mathrm{t}_{10}\end{array}$} \\
\hline ia 1 & Dia 2 & Analista A & Analista B & \\
\hline 11 & 0,00 & 1,50 & 0,22 & $2,23^{\mathrm{a}}$ \\
\hline \multicolumn{5}{|c|}{${ }^{\mathrm{a}}$ valor tabelado de $\mathrm{t}$ (teste bilateral, $\mathrm{p}<0,05$ ). } \\
\hline \multicolumn{5}{|c|}{$\begin{array}{l}\text { temperaturas de } 4{ }^{\circ} \mathrm{C} \text { e } 25{ }^{\circ} \mathrm{C} \text { foram de } 10,18 \\
( \pm 0,17) \mu \mathrm{g} \cdot \mathrm{mL}^{-1} \text { e } 10,17( \pm 0,16) \mu \mathrm{g} . \mathrm{mL}^{-1} \text {, respectivamen- } \\
\text { te. Amudança da fonte fornecedora do metanol resultou em } \\
\text { concentrações médias de } 9,97( \pm 0,18) \mu \mathrm{g} . \mathrm{mL}^{-1} \text { para o tipo } \\
\text { A e } 10( \pm 0,17) \mu \mathrm{g} \cdot \mathrm{mL}^{-1} \text { para o tipo B. Entre todos os resul- } \\
\text { tados experimentais o valor máximo do coeficiente de va- } \\
\text { riação encontrado foi } 1,77 \% \text {. De acordo com o teste } t \text { de } \\
\text { Student (Tabela VI) não há diferença estatisticamente sig- } \\
\text { nificativa entre os resultados obtidos, quando da alteração } \\
\text { na temperatura de análise ou na fonte fornecedora do } \\
\text { solvente durante as análises realizadas, ou seja, no nível de } \\
95 \% \text { de confiança as variações dos parâmetros analisados } \\
\text { não influenciam a quantificação do ácido úsnico. }\end{array}$} \\
\hline
\end{tabular}

TABELA VI - Teste $t$ de Student aplicado aos parâmetros analisados para robustez do método de dosagem do ácido úsnico em espectroscopia UV

\section{Limites de Detecção (LD) e Quantificação (LQ)}

O método demonstrou ser sensível a pequenas concentrações tendo como valor de LQ $1,13 \mu \mathrm{g} \cdot \mathrm{mL}^{-1}$, o qual

TABELA V - Resultados da exatidão para três níveis de concentrações diferentes de ácido úsnico

\begin{tabular}{lrcrrrr}
\hline $\begin{array}{l}\text { Concentração de ácido úsnico }\left(\mu \mathrm{g} . \mathrm{mL}^{-1}\right) \\
\text { Teórica }\end{array}$ & \multicolumn{3}{c}{ Experimental } & & Média & Dados estatísticos \\
\hline 5 & 4,90 & 4,93 & 5,21 & 5,01 & 0,17 & C.V. $(\%)$ \\
10 & 9,93 & 10,12 & 10,20 & 10,08 & 0,14 & 3,41 \\
15 & 15,07 & 14,74 & 15,24 & 15,02 & 0,25 & 1,38 \\
\hline
\end{tabular}

*D.P. $=$ desvio padrão; C.V.= coeficiente de variação. 
corresponde a $11 \%$ da concentração teste. No entanto o LD encontrado foi de $0,34 \mu \mathrm{g} \cdot \mathrm{mL}^{-1}$, concentração esta equivalente a apenas $3,4 \%$ da concentração teste.

\section{Aplicação do Método: Doseamento de Ácido Úsnico em Formas Lipossomais}

O doseamento do ácido úsnico em lipossomas foi de $96,8 \%( \pm 0,2)$. Ao comparar os resultados obtidos com métodos analíticos para determinação de ácido úsnico por CLAE, o método espectrofotométrico proposto apresenta capacidade similar de determinar o analito em questão, uma vez que a faixa de linearidade do método desenvolvido, pode ser considerada equivalente àquela dos métodos CLAE, utilizados para microesferas $\left(1-10 \mu \mathrm{g} \cdot \mathrm{mL}^{-1}\right)$ (Ribeiro-Costa et al., 2004) e nanocápsulas $\left(2-20 \mu \mathrm{g} . \mathrm{mL}^{-1}\right)$ (Santos et al., 2005). Além disso, outro método CLAE associado a detector UV com arranjo de diodos para doseamento do ácido úsnico em matéria-prima e produtos acabados, apresentou resposta linear entre 1,4 e $570 \mu \mathrm{g} \cdot \mathrm{mL}^{-1} \mathrm{e}$ um limite de detecção de $0,4 \mu \mathrm{g} \cdot \mathrm{mL}^{-1}$ (Ji, Khan, 2005), valor este próximo do limite de detecção encontrado no presente trabalho $\left(\mathrm{LD}=0,34 \mu \mathrm{g} \cdot \mathrm{mL}^{-1}\right)$.

\section{CONCLUSÃO}

Um método analítico espectrofotométrico foi proposto e validado para doseamento do ácido úsnico em $280 \mathrm{~nm}$, demonstrando ser simples, rápido, preciso, exato e reprodutível. A análise de variância foi utilizada como ferramenta para análise da linearidade, precisão e exatidão do método. O método foi aplicado para quantificar o ácido úsnico encapsulado em formas lipossomais, após desestruturação dos lipossomas com solvente, sem interferência dos constituintes da formulação. O método analítico espectrofotométrico-UV constitui, portanto, uma ferramenta alternativa, útil, de baixo custo e fácil execução na rotina de controle de qualidade do ácido úsnico como matéria-prima e em formas farmacêuticas tais como lipossomas.

\section{ABSTRACT}

\section{Validation of a UV-spectrophotometric analytical method for the determination of usnic acid in liposomes}

The secondary lichen metabolite usnic acid [2,6-diacetyl7,9-dihydroxy-8,9b-dimethyl-1,3(2H,9bH)-dibenzofuran] has demonstrated pharmacological potential activities such as antitumor, antimicrobial, antiviral, antiproliferative, and anti-inflammatory. Liposomes are vesicles composed of phospholipid bilayers surrounding aqueous compartments and they have been used as colloidal drug carriers. The aim of this study was to develop and validate a quantitative $U V$ spectrophotometric method for determination of usnic acid in liposomal formulations. The validation parameters were assessed according to The International Conference on Harmonization (ICH) and American Pharmacopoeia guidelines. The linearity range was of 3-15 $\mu \mathrm{g} . \mathrm{mL}^{-1}$, regression equation: absorbance $=0.070 \times U A$ concentration $\left(\mu \mathrm{g} \cdot \mathrm{mL}^{-1}\right)+0.013$, and $r=0.9997$. The repeatability (relative standard deviation) of the method was $1.96 \%$ and intermediate precision indicated that the difference among mean was statistically insignificant ( $P$ $<0.05)$. The accuracy revealed a mean percentage recovery of $100.4 \%$ of usnic acid. The method was robust for the variation of temperature and solvent. The detection and quantization limits were found to be 0.34 and 1.13 $\mu \mathrm{g} . m L^{-1}$, respectively. The content of usnic acid in liposomes was of $96.8 \%( \pm 0.2)$. The proposed method is accurate, precise and reproducible for estimation of usnic acid as raw material and in pharmaceutical dosage forms such as liposomes.

UNITERMS: Usnic acid/determination. Liposomes. Ultraviolet Spectrophotometry. Quantitative analysis/ method's validation.

\section{AGRADECIMENTOS}

Os autores agradecem ao Conselho Nacional de Desenvolvimento Científico e Tecnológico $(\mathrm{CNPq} / \mathrm{MCT})$ pelo apoio financeiro concedido a NSSM (Processo n ${ }^{\circ} 479979 / 01-4$ ) e ao Ministério da Ciência e Tecnologia através da Rede Nacional de Nanobiotecnologia (Nanobiotec)-MCT/CNPq. M.P.S.M. agradece à Coordenação de Aperfeiçoamento de Pessoal de Nível Superior (CAPES) e M.C.B.L. agradece à Fundação de Amparo à Ciência e Tecnologia do Estado de Pernambuco (FACEPE), pelas bolsas de mestrado.

\section{REFERÊNCIAS BIBLIOGRÁFICAS}

CLOTHIER, Jr., J. G.; CARLSON, J. R.; COLWELL, D. J. Antiperspirant and deodorant compositions containing a low molecular weight polyethylene gellant. US Patente No. 6.375.938, Abril, 2002.

COCCHIETTO, M.; SKERT, N.; NIMIS, P.L.; SAVA, G. A review on usnic acid, an interesting natural compound. Naturwissenschaften, Berlin, v.89, n.4, p.137-146, 2002. 
ELLIOTT, D. L.; COLWELL, D. J.; SANE, J. N.; VU, T.M.; GALANTE, C. L. Multi-portion antiperspirant composition. U.S. Patente No. 6.936.242, Agosto, 2005.

FERRARI, G.; GHIONE, M.; GHIRARDI, P. Composition containing usnic acid or its derivatives for the therapeutical control of dental caries, particularly for the treatment of the cariogenic dental plaque. Patente No. ZA8704549, Março, 1988.

GHIONE, M.; PARRELLO, D.; GRASSO, L. Usnic acid revisited, its activity on oral flora. Chemioterapia: Int. J. Medit. Soc. Chemother, Florence, v.7, n.5 p.302-305, 1988.

GRASSO, L.; GHIRARDI, P.E.; GHIONE, M. Usnic acid, a selective antimicrobial agent against Streptococcus mutans: a pilot clinical study. Cur. Ther. Res., Amsterdam, v.45, n.6, p.1067-1070, 1989.

GROSZ, R.; MOLONEY, M. J.; KLEINRATH, R.; SOLAN, J. L.; VAN DER LEE, H.; GALANTE, C.L. Methods of manufacturing personal care products. US Patente, No. 6.838.032, Janeiro, 2005.

MERCK INDEX. Encyclopedia of chemical, drugs and biologicals. 12 ed. New Jersey: Merck, 1995. p.9806.

ICH Q2(R1). Validation of Analytical Procedures: Text and Methodology. Disponível em: <http://www.ich.org/LOB/ media/MEDIA417.pdf $>$. Acesso em: 20 mar. 2007.

INGÓLFSDÓTTIR, K.; CHUNG, G.A.C.; SKÚLASON, V.; GISSURARSON, S.R.; VILHELMSDÓTTIR, M. Antimycobacterial activity of lichen metabolites in vitro. Eur. J. Pharm. Sci., Amsterdam, v.6, n.2, p.141-144, 1998.

JI, X.; KHAN, I.A. Quantitative determination of usnic acid in usnea lichen and its products by reversed-phase liquid chromatography with photodiode array detector. J. AOAC Int., Gaithersburg, v.88, n.5, p.1265-1268, 2005.

LASIC, D.D. Liposomes: From physics to application. Amsterdam: Elsevier, 1993. p.3-7.

LAUTERWEIN, M.; OETHINGER, M.; BELSNER, K.; PETERS, T.; MARRE, R. In vitro activities of the lichen secondary metabolites vulpinic acid, (+)-usnic acid, and (-)-usnic acid against aerobic and anaerobic microorganisms. Antimicrob. Agents Chemother., Bethesda, v.39, n.11, p.2541-2543, 1995.
LODETTI, G.; GOGOLA, P.; BERTASI, B.; D'AMBROSCA, F.; PONCHIO, G.; FISCHBACH, M.; LOSIO, M.N. Valutazione in vitro della citotossità indotta da prodotti per l'igiene orale contenenti acido usnico, agente antimicrobiano naturale estratto dai licheni. G. Ital. Microbiol. Med., Milano, v.4, n. , p.67-72, 2000.

NEFF, G. W.; REDDY, K. R.; DURAZO, F.A.; MEYER, D.; MARRERO, R; KAPLOWITZ, N. Severe hepatotoxicity associated with the use of weight loss diet supplements containing ma huang or usnic acid. Letters to the Editor/ J. Hepatol., v.41, p.1061-1067, 2004.

PÉREZ-LOZANO, P.; GARCÍA-MONTOYA, E.; ORRIOLS, A.; MIÑARRO, M.; TICÓ, J.R.; SUÑÉNEGRE, J.M. Development and validation of a new HPLC analytical method for the determination of alprazolam in tablets. J. Pharm. Biomed. Anal., Amsterdam, v.34, n.5, p.979-987, 2004.

RIBEIRO-COSTA, R.M.; ALVES, A.J.; SANTOS, N.P.; NASCIMENTO, S.C.; GONÇALVES, E.C.P.; SILVA, N.H.; HONDA, N.K.; SANTOS-MAGALHÃES, N.S. In vitro and in vivo properties of usnic acid encapsulated into PLGA-microspheres. J. Microencapsul., London, v.21, n.4, p.371-384, 2004.

SANTOS, N.P.; NASCIMENTO, S.C.; SILVA, J.F.; PEREIRA, E.C.G.; SILVA, N.H.; HONDA, N.K.; SANTOS-MAGALHÃES, N.S. Usnic acid-loaded nanocapsules: an evaluation of cytotoxicity. J. Drug Del. Sci. Technol., Paris, v.15, n.5, p.355-361, 2005.

SCIRPA, P.; SCAMBIA, G.; MASCIULLO, V.; BATTAGLIA, F.; FOTI, E.; LOPEZ, R.; VILLA, P.; MALECOE, M.; MANCUSO, S. Terapia adiuvante com um preparato a base di zinco solfato e acido úsnico delle lesioni genitali da Human Papilloma Vírus (HPV) dopo trattamento chirurgico distruttivo. Min. Ginecol., Torino, v.51, n.6, p.255-260, 1999.

TAKANI, M.; YAJIMA, T.; MASUDA, H.; YAMAUCHI, O. Spectroscopic and structural characterization of copper (II) and palladium (II) complexes of a lichen substance: usnic acid and its derivatives. Possible forms of environmental metals retained in lichens. J. Inorg. Biochem., New York, v.91, n.1, p.139-150, 2002.

UNITED States Pharmacopoeia. 26. ed. Rockville: United States Pharmacopoeial Convention, 2003. p.2439-2442. 
VENKATARAMANA, D.; KRISHNA, D.R. Highperformance liquid chromatographic determination of usnic acid in plasma. J. Chromatogr. Biomed. Appl., Amsterdam, v.575, n.1, p.167-170, 1992.

Recebido para publicação em 02 de agosto de 2006 Aceito para publicação em 24 de julho de 2008 